\title{
Prevention of dry socket with metronidazole
}

\author{
The prevention of 'dry socket' with topical metronidazole in general dental practice
}

D. Reekie, P. Downes, C. V. Devlin, G. M. Nixon and H. Devlin Br Dent J 2005; 200: 210-213

\begin{abstract}
Objective
The purpose of the study was to determine if the intra-alveolar application of topical metronidazole gel could reduce the incidence of alveolar osteitis (dry socket) following routine tooth extraction in molar and premolar extraction sites.

Design

This was a multicentre, double blind, randomised, placebo-controlled clinical trial. A total of 302 patients took part, of which 23 returned with alveolar osteitis. Of these, eight had received the metronidazole gel and 15 the placebo.

Setting

The study was carried out in three general dental practices by general dental practitioners working in England over the period 2000-2003. Main outcome measures

Following extraction of either a molar or premolar tooth, either a 25\% metronidazole gel or KY Jelly was syringed gently into the socket. A painful post operative complication was recorded if either a dry socket was present or the patient returned with pain.

Results and conclusions

The difference in the incidence of alveolar osteitis between the placebo and the active gel groups was not significant and it was concluded that $25 \%$ topical metronidazole gel was not effective in reducing the incidence of alveolar osteitis. It was found that the incidence of alveolar osteitis reduced with increasing age and was more likely to occur in a patient with a previous history of the condition.
\end{abstract}

\section{IN BRIEF}

- A previous history of alveolar osteitis predisposed the patient to painful post-operative complications following molar or premolar tooth extraction.

- Topical metronidazole gel was ineffective in preventing either alveolar osteitis or the patient returning with pain.

- Complications following tooth extraction are significantly less in those aged 50 years and over compared with those less than 50 years of age.

\section{COMMENT}

Dry socket or acute alveolar osteitis is a common and often very painful and distressing condition for a patient who has recently undergone a tooth extraction.

This paper reports on a multicentre, double blind, randomised, placebo controlled clinical trial to determine if the local application of a metronidazole gel into the tooth socket postextraction could reduce the occurrence of dry sockets.

The study was carried out entirely in a general dental practice setting by four general dental practitioners over a three year period. Three hundred and two patients were entered into the study resulting in 23 dry sockets.

The rationale for using a topical antibiotic was based on previous research which had shown that systemic metronidazole could significantly reduce the incidence of dry socket when administered post extraction and infection of the socket being an important aetiologic factor. Previous studies had also shown that topical clindamycin and tetracycline were effective. Predisposing factors such as smoking, the oral contraceptive pill, poor oral hygiene, surgical trauma and a history of previous dry sockets have been shown to play a role.

Twenty-three patients in the study developed a dry socket, eight had had metronidazole placed in the sockets and 15 a placebo of KY Jelly. Analysis of the results indicated no statistical difference between the two groups but did show a reduced incidence with increasing age and was more likely to occur in people with a previous history of dry sockets.

Smokers were not excluded but simply asked to refrain from smoking during the healing period. This in my opinion of dealing with smokers is very unreliable and many will continue smoking even in cases of recently diagnosed malignancy.

It is commendable to see general dental practitioners taking an active part in research and this should be encouraged

R. Lloyd, Consultant Oral and Maxillofacial Surgeon, Manchester Royal Infirmary doi: 10.1038/sj.bdj.4813254 